\title{
Performance of the Smart Antenna Aided Generalized Multicarrier DS-CDMA Downlink Using Both Time-Domain Spreading and Steered Space-Time Spreading
}

\author{
Bin $\mathrm{Hu}$, Lie-Liang Yang and Lajos Hanzo \\ School of ECS, University of Southampton, SO17 1BJ, UK. \\ Tel: +44-23-8059 3125, Fax: +44-23-8059 4508 \\ Email: bh202r,1ly,lh@ecs.soton.ac.uk; http://www-mobile.ecs.soton.ac.uk
}

\begin{abstract}
In this contribution a generalized MC DS-CDMA system invoking smart antennas for improving the achievable performance in the downlink of the system is studied, which is capable of minimizing the downlink interference inflicted upon co-channel mobiles, while achieving frequency, time and spatial diversity. In the MC DS-CDMA system considered the transmitter employs multiple antenna arrays and each of the antenna arrays consists of several antenna elements. More specifically, the space-time transmitter processing scheme considered is based on the principles of Steered Space-Time Spreading (SSTS). Furthermore, the generalized MC DS-CDMA system employs time and frequency (TF)-domain spreading, where a user-grouping technique is employed for reducing the effects of multiuser interference.
\end{abstract}

\section{INTRODUCTION}

In recent years numerous research contributions have appeared on the topic of Multi-Carrier Direct Sequence Code Division Multiple Access (MC DS-CDMA), which constitutes an attractive scheme [1], [2], [3], [4], based on a combination of DS-CDMA and OFDM. In this contribution, we discuss the generalized MC DS-CDMA system investigated in [5], [6], where it has been shown that the generalized MC DS-CDMA scheme includes the subclasses of both multitone DSCDMA [3] and orthogonal MC DS-CDMA [4] as special cases. On the other hand, smart antennas have been used for improving the performance of wireless systems [7], [8]. Various transmit diversity (TD) schemes have been proposed in the literature for the downlink [9], [10], [11] for the sake of mitigating the performance degradation inflicted by multipath fading. On the other hand, beamforming [7], [11] constitutes an effective technique of reducing the multiple-access interference (MAI), where the antenna gain is increased in the direction of the desired user, whilst reducing the gain towards the interfering users. Using the antenna arrays shown in Fig.1, both TD and beamforming can be implemented at the base station (BS).

The novelty of this paper is that we combine the above-mentioned generalized MC DS-CDMA system [5], [6] with smart antennas for the sake of improving the performance in the downlink of the system by minimizing the downlink interference inflicted upon co-channel mobiles, while achieving frequency, time and spatial diversity. The achievable performance improvements are a function of both the antenna spacing and the specific techniques used for attaining beamforming. Using the antenna arrays shown in Fig.1, a space-time trans-

The financial support of the EPSRC, UK and the EU under the auspices of the Phoenix and Newcom projects is gratefully acknowledged. mitter processing scheme based on the principles of Steered SpaceTime Spreading (SSTS) is proposed and characterized, in order to enhance the attainable performance of the generalized MC DS-CDMA system. Several different antenna array models have been investigated. Furthermore, the generalized MC DS-CDMA using TF-domain spreading has been investigated, while employing a user-grouping technique for reducing the multiuser interference imposed.

The rest of this paper is organized as follows. In Section II the philosophy of the downlink SSTS-aided generalized Multicarrier DSCDMA system is described and characterized. Then, Section III considers the achievable system capacity improvement of generalized MC DS-CDMA using SSTS and TF-domain spreading. The attainable performance of these schemes is studied and compared in Section IV, again in the context of the generalized MC DS-CDMA system concept advocated. Finally, our conclusions are offered in Section V.

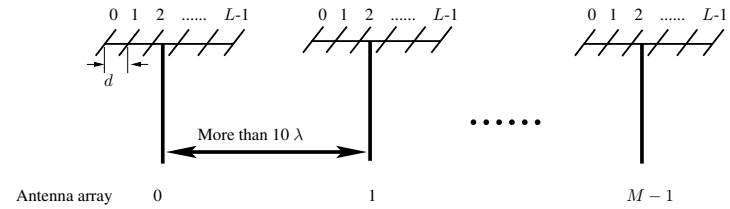

Fig. 1. Multiple transmitter antenna configuation to be used in the generalized MC DS-CDMA system considered.

\section{Steered Space-Time Spreading - Downlink}

In [12], a hybrid downlink technique designed for achieving both transmit diversity and transmit beamforming was proposed for DSCDMA, which is referred to as steered space-time spreading (SSTS). By contrast, in this contribution we extend our investigations to the generalized MC DS-CDMA system of [5], [6]. It can be shown that TF-domain spreading can be employed for increasing the number of users supported, while the beamforming scheme invoked is capable of reducing the increased interference imposed by the increased number of users facilitated by TF-domain spreading.

\section{A. Transmitter Model - SSTS}

The system considered in this section is a generalized MC DSCDMA scheme [5], [6] using $U \cdot V$ number of subcarriers. The transmitter schematic of the $k$ th user is shown in Fig.2, where a block of $U \cdot M$ data bits each having a bit duration of $T_{b}$ is Serial-Parallel (S-P) 


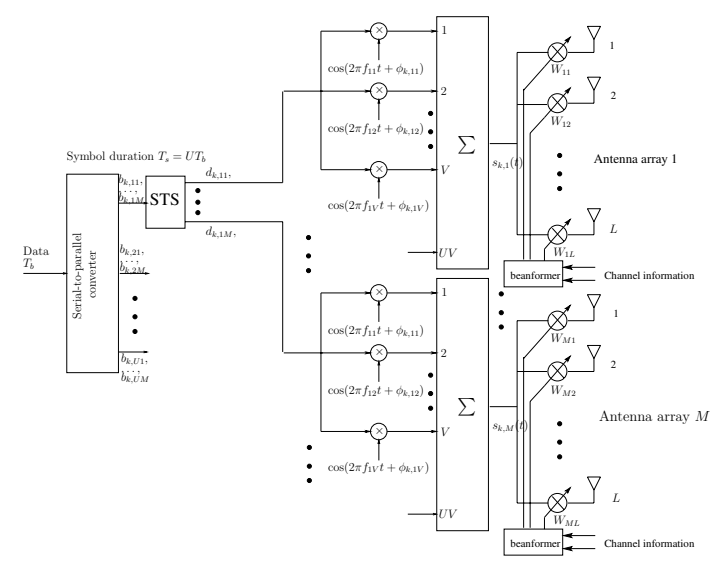

Fig. 2. The $k$ th user's transmitter schematic for the downlink of the SSTS assisted generalized multicarrier DS-CDMA system.

converted to $U$ parallel sub-blocks. The new bit duration of each substream, which we refer to as the symbol duration, becomes $T_{s}=U T_{b}$. After the STS procedure described in [13], which spreads the signals to be transmitted to multiple antennas with the aid of $M$ orthogonal spreading codes $\left\{c_{k, 1}(t), c_{k, 2}(t), \ldots, c_{k, M}(t)\right\}, k=1,2, \ldots, K$, the $U M$ outputs of the $U$ number of STS blocks are multicarrier modulated by a group of subcarrier frequencies $\left\{f_{u 1}, f_{u 2}, \ldots, f_{u V}\right\}$ using Binary Phase Shift Keying (BPSK) and then forwarded to the transmitter's beamformer. For the case of $M=2$ beamformer arrays we have two orthogonal spreading codes for the $k$ th user, which are

$$
\begin{aligned}
& c_{k, 1}(t)=[1,1] c_{k}(t)=\left[c_{k}(t), c_{k}(t)\right], \\
& c_{k, 2}(t)=[1,-1] c_{k}(t)=\left[c_{k}(t),-c_{k}(t)\right]
\end{aligned}
$$

The symbol duration of the space-time spread (STS) signals is $M T_{s}$, and the length of the orthogonal codes is $M T_{s} / T_{c}=M N_{e}$, where $N_{e}=T_{s} / T_{c}$ and $T_{c}$ represents the chip-duration of the orthogonal STS codes. Finally, according to the $k$ th user's Frequency-Domain Channel Transfer Function (FDCHTF), the $U M V$ signals of the $k$ th user are weighted by the transmit weight vector $\mathbf{w}_{v, m}^{(k)}$ determined for the $v$ th subcarrier of the $k$ th user, which is generated for the $m$ th array.

The general form of the $k$ th user's transmitted signal corresponding to the $M$ transmit antennas can be expressed as

$$
\begin{aligned}
\mathbf{s}_{k}(t)= & \sum_{u=1}^{U} \sum_{v=1}^{V} \sqrt{\frac{2 P_{k}}{V L} \frac{1}{M \cdot M}} \mathbf{W}_{v}^{(k)} \mathbf{B}_{k u} \mathbf{c}_{k} \\
& \times \cos \left(2 \pi f_{u v} t+\phi_{k, u v}\right),
\end{aligned}
$$

where $P_{k} / V$ represents the transmitted power of each subcarrier, while the factor of $M \cdot M$ represents STS using $M$ orthogonal codes and $M$ transmit beamformer antenna arrays, while the factor $L$ indicates that each of the antenna arrays has $L$ elements. In (2) $\mathbf{c}_{k}=$ $\left[c_{k, 1}(t), c_{k, 2}(t), \ldots, c_{k, M}(t)\right]^{T}$ is an $M$-dimensional vector constituted by the orthogonal STS codes, while $\mathbf{B}_{k u}$ is an $(M \times M)$ dimensional matrix mapping the data from the $u$ th sub-block data bits, which are output by the S-P converter, to the $M$ transmit arrays, according to the requirements of the STS scheme [13]. For $M=2$, the

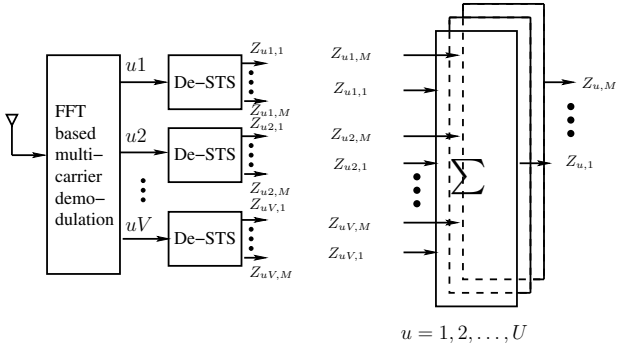

Fig. 3. The receiver schematic of the generalized multicarrier DS-CDMA system associated with SSTS.

corresponding $\mathbf{B}_{k u}$ matrix is given by [13]

$$
\left(\begin{array}{cc}
b_{k, u 1} & b_{k, u 2} \\
b_{k, u 2} & -b_{k, u 1}
\end{array}\right), u=1,2, \ldots, U .
$$

In (2), $\mathbf{W}_{v}^{(k)}$ is the weight matrix, which can be expressed as

$$
\mathbf{W}_{v}^{(k)}=\left[\begin{array}{cccc}
\left(\mathbf{w}_{v, 0}^{(k)}\right)^{*} & \mathbf{0} & \ldots & \mathbf{0} \\
\mathbf{0} & \left(\mathbf{w}_{v, 1}^{(k)}\right)^{*} & \ldots & \mathbf{0} \\
\vdots & \vdots & \ddots & \vdots \\
\mathbf{0} & \mathbf{0} & \ldots & \left(\mathbf{w}_{v,(M-1)}^{(k)}\right)^{*}
\end{array}\right]
$$

where $\mathbf{0}=[0,0, \ldots, 0]^{T}$ is an $L$-dimensional vector, $\mathbf{w}_{v, m}^{(k)}$ is the $L$ dimensional weight vector for the $m$ th beamformer antenna array and the $v$ th subcarrier of the $k$ th user.

Equation (2) represents the general form of the transmitted signals using SSTS, regardless of the value of $M$. However, in this contribution we investigate MC DS-CDMA systems using two antenna arrays. Specifically, for the case of $M=2$, the MC DS-CDMA signals transmitted by antenna array 1 and 2 can be simply expressed as

$$
\begin{aligned}
\mathbf{s}_{k}(t)= & \sqrt{\frac{2 P_{k}}{4 V L}}\left(\begin{array}{l}
\sum_{u=1}^{U} \sum_{v=1}^{V}\left[c_{k, 1} b_{k, u 1}+c_{k, 2} b_{k, u 2}\right] \mathbf{w}_{v, 0}^{(k)} \\
\sum_{u=1}^{U} \sum_{v=1}^{V}\left[c_{k, 1} b_{k, u 2}-c_{k, 2} b_{k, u 1}\right] \mathbf{w}_{v, 1}^{(k)}
\end{array}\right) \\
& \times \cos \left(2 \pi f_{u v} t+\phi_{k, u v}\right) .
\end{aligned}
$$

\section{B. Receiver Model-SSTS}

The Spatio-Temporal (ST) Channel Impulse Response (CIR) vector $h_{u v, m}^{(k)}$ between the $u v$ th subcarrier of the $k$ th user and the $m$ th antenna array can be expressed as

$$
\begin{aligned}
\mathbf{h}_{u v, m}^{(k)}(t) & =\mathbf{a}_{u v, m}^{(k)}(t) \delta\left(t-\tau_{k}\right) \\
& =\left[a_{u v, m 0}^{(k)}(t), \ldots, a_{u v, m(L-1)}^{(k)}(t)\right]^{T} \delta\left(t-\tau_{k}\right),
\end{aligned}
$$

which is an $L$-dimensional vector, where $\tau_{k}$ is the signal's delay, $a_{u v, m l}^{(k)}(t)$ is the CIR with respect to the $u v$ th subcarrier of the 1st user and the $l$ th element of the $m$ th antenna array. Based on the assumption 
that the array elements are separated by half a wavelength, i.e. that we have $d=\lambda / 2$, we can simplify $\mathbf{a}_{u v, m}^{(k)}(t)$ to

$$
\mathbf{a}_{u v, m}^{(k)}(t)=\alpha_{u v, m}^{(k)}(t) \mathbf{d}_{m}^{(k)},
$$

where $\alpha_{u v, m}^{(k)}(t)$ is the Rayleigh faded envelope's amplitude, $\mathbf{d}_{m}^{(k)}=$ $\left[1, \exp \left(j\left[\pi \sin \left(\psi_{m}^{(k)}\right)\right]\right), \ldots, \exp \left(j\left[(L-1) \pi \sin \left(\psi_{m}^{(k)}\right)\right]\right)\right]^{T}$, and $\psi_{m}^{(k)}$ is the average Direction-Of-Arrival (DOA). Assuming that $K$ user signals in the form of (4) are transmitted synchronously over Rayleigh fading channels, the received complex low-pass equivalent signal can be expressed as

$$
r(t)=\sum_{k=1}^{K} r_{k}(t)+n(t)
$$

where $n(t)$ is the AWGN having a zero mean and a variance of $\mathrm{E}\left[n\left(t_{1}\right) n^{*}\left(t_{2}\right)\right]=2 N_{0} \delta\left(t_{1}-t_{2}\right)$, and $N_{0}$ represents the doublesided power spectral density of a complex valued low-pass-equivalent AWGN signal. In (8) $r_{k}(t)$ represents the $k$ th user's transmitted signal received by the 1 st user, which can be expressed as

$$
\begin{aligned}
r_{k}(t)= & \sum_{u=1}^{U} \sum_{v=1}^{V} \sqrt{\frac{2 P_{k}}{V L} \frac{1}{M M}} \mathbf{c}_{k}^{T} \mathbf{B}_{k u}^{T}\left(\mathbf{W}_{v}^{(k)}\right)^{T} \mathbf{h}_{u v}^{(1)}(t) \\
& \times \cos \left(2 \pi f_{u v} t+\phi_{k, u v}\right)
\end{aligned}
$$

where $\mathbf{h}_{u v}^{(1)}(t)=\left[\left(\mathbf{h}_{u v, 0}^{(1)}(t)\right)^{T}, \ldots,\left(\mathbf{h}_{u v,(M-1)}^{(1)}(t)\right)^{T}\right]^{T}$ is a $M L$ dimensional ST-CIR vector corresponding to the first user.

The receiver front-end is shown in Fig.3. After multicarrier demodulation, the $U V$ number of parallel streams corresponding to the signals transmitted on the $U V$ subcarriers are space-time despread using the approach of [13], in order to obtain $M$ separate variables, $\left\{z_{u v, 1}, z_{u v, 2}, \ldots, z_{u v, M}\right\}$, corresponding to the $M$ number of data bits transmitted on the $u v$ th stream, where we have $u=$ $1,2, \ldots, U ; v=1,2, \ldots, V$, respectively. For the case of $M=2$, let $y_{u v, 1}, y_{u v, 2}$ represent the correlator's output variables corresponding to the first two data bits transmitted on the $u v$ th subcarrier. Since orthogonal multicarrier signals, orthogonal STS codes, synchronous transmission of the $K$ user signals as well as slowly flat-fading of each subcarrier are assumed, there is no interference between the different users and the different subcarrier signals. Therefore, $y_{u v, 1}, y_{u v, 2}$ can be expressed as

$$
\begin{aligned}
y_{u v, 1}= & \sqrt{\frac{2 P}{V L}} T_{s}\left[\left(\mathbf{w}_{v, 0}^{(1)}\right)^{H} \mathbf{a}_{u v, 0}^{(1)} b_{1, u 1}\right. \\
& \left.+\left(\mathbf{w}_{v, 1}^{(1)}\right)^{H} \mathbf{a}_{u v, 1}^{(1)} b_{1, u 2}\right]+n_{u v, 1}, \\
y_{u v, 2}= & \sqrt{\frac{2 P}{V L}} T_{s}\left[\left(\mathbf{w}_{v, 0}^{(1)}\right)^{H} \mathbf{a}_{u v, 0}^{(1)} b_{1, u 2}\right. \\
& \left.-\left(\mathbf{w}_{v, 1}^{(1)}\right)^{H} \mathbf{a}_{u v, 1}^{(1)} b_{1, u 1}\right]+n_{u v, 2},
\end{aligned}
$$

where $n_{u v, i}, i=1,2$ is due to the AWGN processing, which is a complex Gaussian distributed variable having zero mean and a variance of $2 N_{0} T_{s}$. In (10) and (11), $\mathbf{w}_{v, m}^{(1)}, m=0,1$, represents the weight matrix of the desired user on the $m$ th antenna array, which is generated by the MRC beamformer with the aid of the channel state information.
It can be shown that we have $\mathbf{w}_{v, m}^{(1)}=\mathbf{d}_{m}^{(1)}, m=1,2$, where $\mathbf{d}_{m}^{(1)}$ is given in (7). Consequently, (10) and (11) can be simplified to

$$
\begin{aligned}
& y_{u v, 1}=\sqrt{\frac{2 P L}{V}} T_{s}\left[\alpha_{u v, 0} b_{1, u 1}+\alpha_{u v, 1} b_{1, u 2}\right]+n_{u v, 1}, \\
& y_{u v, 2}=\sqrt{\frac{2 P L}{V}} T_{s}\left[\alpha_{u v, 0} b_{1, u 2}-\alpha_{u v, 1} b_{1, u 1}\right]+n_{u v, 2} .
\end{aligned}
$$

Assuming that the receiver has perfect knowledge of the fading parameters of $\alpha_{u v, m}, m=1,2$, the decision variables corresponding to the data bits $b_{1, u m}, m=1,2$ associated with the $u v$ th subcarrier can be expressed as

$$
\begin{aligned}
z_{u v, 1}= & \sqrt{\frac{2 P L}{V}} T_{s}\left[\left\|\alpha_{u v, 0}\right\|^{2}+\left\|\alpha_{u v, 1}\right\|^{2}\right] b_{1, u 1} \\
& +n_{u v, 1} \alpha_{u v, 0}^{*}-n_{u v, 2}^{*} \alpha_{u v, 1}, \\
Z_{u v, 2}= & \sqrt{\frac{2 P L}{V}} T_{s}\left[\left\|\alpha_{u v, 0}\right\|^{2}+\left\|\alpha_{u v, 1}\right\|^{2}\right] b_{1, u 2} \\
& +n_{u v, 1} \alpha_{u v, 1}^{*}+n_{u v, 2}^{*} \alpha_{u v, 0},
\end{aligned}
$$

for $u=1,2, \ldots, U ; v=1,2, \ldots, V$.

Finally, after combining the replicas of the same signal transmitted on the $V$ subcarriers, the decision variables corresponding to the two bits in the $u$ th sub-block can be expressed as

$$
z_{u, 1}=\sum_{v=1}^{V} z_{u v, 1} \text { and } z_{u, 2}=\sum_{v=1}^{V} Z_{u v, 2}
$$

for $u=1,2, \ldots, U$.

\section{CAPACITy EXTENSION USING TIME-FREQUENCY-Domain SPREADING - SSTS}

Above the DS spreading used in MC DS-CDMA systems is carried out in the time-domain (T-domain) based on orthogonal WalshHadamard DS-spreading codes. However, as proposed for MC-CDMA schemes in [15], spreading in the F-domain may also be employed to exploit the independent fading of the subcarriers in the F-domain. Hence in the generalized MC DS-CDMA scheme, the transmitted data stream can be spread in both the T-domain and the F-domain in order to support more users, while achieving a high frequency diversity gain [16]. The resultant bandwidth is the same as that of the MC DSCDMA scheme employing time-domain spreading only, while the total number of users supported is $V \mathcal{K}_{\max }=V N_{e}$, which is $V$ times the number of users supported by the MC DS-CDMA scheme employing time-domain only spreading.

The transmitter schematic of the broadband MC DS-CDMA system using TF-domain spreading is similar to that seen in Fig.2, except that the $V$-depth F-domain repetition of Fig.2 is now replaced by the F-domain spreading associated with an orthogonal spreading code of length $V$. Accordingly, let $\left\{c_{k}^{\prime}[0], c_{k}^{\prime}[1], \ldots, c_{k}^{\prime}[V-1]\right\}$ be the $k$ th user's orthogonal F-domain spreading code in discrete form. Following the approach of Section II, the terms $y_{u v, 1}, y_{u v, 2}$, can be expressed as

$$
y_{u v, 1}=\sum_{k=1}^{K^{\prime}} \sqrt{\frac{2 P_{k}}{V L}} T_{s}\left[\left(\mathbf{w}_{v, 0}^{(k)}\right)^{H} \mathbf{a}_{u v, 0}^{(1)} b_{k, u 1}\right.
$$




$$
\begin{aligned}
& \left.+\left(\mathbf{w}_{v, 1}^{(k)}\right)^{H} \mathbf{a}_{u v, 1}^{(1)} b_{k, u 2}\right] c_{k}^{\prime}[v-1]+n_{u v, 1}, \\
y_{u v, 2}= & \sum_{k=1}^{K^{\prime}} \sqrt{\frac{2 P_{k}}{V L}} T_{s}\left[\left(\mathbf{w}_{v, 0}^{(k)}\right)^{H} \mathbf{a}_{u v, 0}^{(1)} b_{k, u 2}\right. \\
& \left.-\left(\mathbf{w}_{v, 1}^{(k)}\right)^{H} \mathbf{a}_{u v, 1}^{(1)} b_{k, u 1}\right] c_{k}^{\prime}[v-1]+n_{u v, 2},
\end{aligned}
$$

where $n_{u v, i}, i=1,2$ is due to the AWGN, which is a complex Gaussian distributed variable having zero mean and a variance of $2 N_{0} T_{s}$ as expressed in (10). In (17), $\mathbf{w}_{v, m}^{(k)}, m=0,1$, represents the weight matrix corresponding to the $k$ th user and the $m$ th transmit antenna array, which is generated by the Maximum Ratio Combining (MRC) based beamformer as shown in section II-B. Consequently, we have $\left(\mathbf{w}_{v, m}^{(k)}\right)=\mathbf{d}_{m}^{(k)}, m=0,1$, in which $\mathbf{d}_{m}^{(1)}$ is shown in (7). In this case, (17) and (18) may be simplified to

$$
\begin{aligned}
y_{u v, 1}= & \sqrt{\frac{2 P L}{V}} T_{s}\left[\alpha_{u v, 0} b_{1, u 1}+\alpha_{u v, 1} b_{1, u 2}\right] c_{k}^{\prime}[v-1] \\
& +n_{u v, 1}+i_{u v, 1}, \\
y_{u v, 2}= & \sqrt{\frac{2 P L}{V}} T_{s}\left[\alpha_{u v, 0} b_{1, u 2}-\alpha_{u v, 1} b_{1, u 1}\right] c_{k}^{\prime}[v-1] \\
& +n_{u v, 2}+i_{u v, 2},
\end{aligned}
$$

where

$i_{u v, 1}=\sum_{k=2}^{K^{\prime}} \sqrt{\frac{2 P_{k}}{V L}} T_{s}\left[\beta_{k, 0} \alpha_{u v, 0} b_{k, u 1}+\beta_{k, 1} \alpha_{u v, 1} b_{k, u 2}\right] c_{k}^{\prime}[v-1]$, $i_{u v, 2}=\sum_{k=2}^{K^{\prime}} \sqrt{\frac{2 P_{k}}{V L}} T_{s}\left[\beta_{k, 0} \alpha_{u v, 0} b_{k, u 2}-\beta_{k, 1} \alpha_{u v, 1} b_{k, u 1}\right] c_{k}^{\prime}[v-1]$, and $\beta_{k, m}=\left(\mathbf{d}_{m}^{(k)}\right)^{H} \mathbf{d}_{m}^{(1)}$. Assuming that the receiver has perfect knowledge of the fading parameters of $\alpha_{u v, m}, m=1,2$, the decision variables corresponding to the data bits $b_{1, u m}, m=1,2$ associated with the $u v$ th subcarrier can be expressed as

$z_{u v, 1}=\sqrt{\frac{2 P L}{V}} T_{s}\left[\left\|\alpha_{u v, 0}\right\|^{2}+\left\|\alpha_{u v, 1}\right\|^{2}\right] b_{1, u 1}+n_{u v, 1}^{\prime}+i_{u v, 1}^{\prime}$,

$z_{u v, 2}=\sqrt{\frac{2 P L}{V}} T_{s}\left[\left\|\alpha_{u v, 0}\right\|^{2}+\left\|\alpha_{u v, 1}\right\|^{2}\right] b_{1, u 2}+n_{u v, 2}^{\prime}+i_{u v, 2}^{\prime}$,

for $u=1,2, \ldots, U ; v=1,2, \ldots, V$, where $n_{u v, 1}^{\prime}=n_{u v, 1} \alpha_{u v, 0}^{*}-$ $n_{u v, 2}^{*} \alpha_{u v, 1}, n_{u v, 2}^{\prime}=n_{u v, 1} \alpha_{u v, 1}^{*}+n_{u v, 2}^{*} \alpha_{u v, 0}, i_{u v, 1}^{\prime}=i_{u v, 1} \alpha_{u v, 0}^{*}-$ $i_{u v, 2}^{*} \alpha_{u v, 1}$, and $i_{u v, 2}^{\prime}=i_{u v, 1} \alpha_{u v, 1}^{*}+i_{u v, 2}^{*} \alpha_{u v, 0}$. Finally, in the context of the single-user correlation based detector, the decision variable $z_{u, 1}$ of the desired user is obtained by combining $\mathbf{z}_{u, 1}=$ $\left[z_{u 1,1}, z_{u 2,1}, \ldots, z_{u V, 1}\right]$ with $\mathbf{c}_{1}^{\prime}=\left[c_{1}^{\prime}[0], c_{1}^{\prime}[1], \ldots, c_{1}^{\prime}[V-1]\right]$, which can be expressed as

$$
\begin{aligned}
z_{u, 1}= & \sqrt{\frac{2 P L}{V}} T_{s}\left[\sum_{v=1}^{V}\left[\left\|\alpha_{u v, 0}\right\|^{2}+\left\|\alpha_{u v, 1}\right\|^{2}\right] b_{1, u 1}\right. \\
& \left.+\mathbf{c}_{1}^{\prime} \mathbf{i}_{u, 1}^{\prime}+\mathbf{c}_{1}^{\prime} \mathbf{n}_{u, 1}^{\prime}\right]
\end{aligned}
$$

where $\mathbf{n}_{u, 1}^{\prime}=\left[n_{u 1,1}^{\prime}, n_{u 2,1}^{\prime}, \ldots, n_{u V, 1}^{\prime}\right]^{T}$ is the $V$-dimensional noise vector, while $\mathbf{i}_{u, 1}^{\prime}=\left[i_{u 1,1}^{\prime}, i_{u 2,1}^{\prime}, \ldots, i_{u V, 1}^{\prime}\right]^{T}$ is the $V$-dimensional interference vector. Then the interference term $\mathbf{c}_{1}^{\prime} \mathbf{i}_{u, 1}^{\prime}$ can be expressed as

$$
\begin{aligned}
\mathbf{c}_{1}^{\prime} \mathbf{i}_{u, 1}^{\prime}= & \sum_{v=1}^{V} \sum_{k=2}^{K^{\prime}} \sqrt{\frac{2 P_{k}}{V L}} T_{s} c_{1}^{\prime}[v-1] c_{k}^{\prime}[v-1] \\
& \times\left[\left(\beta_{k, 0}\left\|\alpha_{u v, 0}\right\|^{2}+\beta_{k, 1}^{*}\left\|\alpha_{u v, 1}\right\|^{2}\right) b_{k, u 1}\right. \\
& \left.+\left(\beta_{k, 1} \alpha_{u v, 0}^{*} \alpha_{u v, 1}-\beta_{k, 0}^{*} \alpha_{u v, 0}^{*} \alpha_{u v, 1}\right) b_{k, u 2}\right]
\end{aligned}
$$

We know from Eq.(21) that multiuser interference is inevitably introduced, since the orthogonality of the F-domain spreading codes cannot be retained over frequency-selective fading channels. However, the desired signal is not interfered by the transmitted signals of the users employing different orthogonal T-domain STS spreading codes, provided that synchronous transmission of all the $K^{\prime} \mathcal{K}_{\max }$ user signals as well as slow flat-fading of each subcarrier are assumed. Only the users sharing the same T-domain STS spreading code with the desired user will impose interference on the desired user. Therefore, the interference $\mathbf{c}_{1}^{\prime} \mathbf{i}_{u, 1}^{\prime}$ can be reduced, if we carefully select the $K^{\prime}-1$ users, namely those which have the lowest interference coefficient with respect to the desired user, from the set of all the $K^{\prime} \mathcal{K}_{\max }$ users for the sake of sharing the same T-domain spreading code with the desired user. Let the interference coefficient be defined as $\sqrt{P_{k}} \beta_{k, 0}$ and $\sqrt{P_{k}} \beta_{k, 1}$, which can be evaluated before transmission based on the assumption that $\mathbf{a}_{u v, m}^{(1)}(t)$ is perfectly estimated. We also assume that $\mathbf{w}_{v, m}^{(k)}=\mathbf{a}_{u v, m}^{(k)}(t)$ is perfectly estimated. Following this usergrouping procedure, the effect of the interfering signals imposed on the desired user's signal becomes less pronounced. Therefore, the achievable BER performance is improved.

\section{PERformance Results}

In this section the BER performance of the downlink of a generalized MC DS-CDMA system employing SSTS and using 32-chip Walsh codes as T-domain spreading sequences as well as 4-chip Walsh codes as F-domain spreading codes is studied. Perfect power control is assumed, implying that $P_{0}=P_{1}=\ldots=P_{K}$.

In Figure 4 we use a $(2 \times 2)$-dimensional antenna array $(M=$ $2, L=2$ ), having an element-spacing of $\lambda / 2$ as well as an arrayspacing of $10 \lambda$. It transpires from Figure 4 that the performance of the system supporting 32 users is similar to that of the system supporting a single user. Recall the assumptions of using orthogonal multicarrier signals, synchronous transmission of the $K$ user signals as well as slow flat-fading of each subcarrier, where no interference is encountered between the 32 users employing different orthogonal 32-chip Walsh codes as T-domain DS spreading codes. In order to extend the usercapacity of the system, TF-domain spreading is employed for the sake of supporting 64 users. Consequently, multiuser interference among the users sharing the same T-domain spreading code is inevitably introduced, since the orthogonality of the F-domain spreading codes cannot be retained, when communicating over frequency-selective fading channels. Then a specific user grouping technique is used to reduce the multiuser interference imposed. Figure 4 shows that the performance of the system supporting 64 users is significantly worse than that supporting a single user or even 32 users, unless employing the proposed user grouping. The system employing the interference coefficient based user grouping technique substantially outperforms the system refraining from user grouping.

Finally, in Figure 5 the performance of the system employing two different types of antenna arrays was compared, namely when using a $(2 \times 2)$-dimensional antenna array $(M=2, L=2)$, and when employing a $(2 \times 3)$-dimensional antenna array $(M=2, L=3)$. 
Observe in Figure 5 that as expected, the system using a $(2 \times 3)$ dimensional antenna array $(M=2, L=3)$ achieves a better performance than the system using a $(2 \times 2)$-dimensional antenna array ( $M=2, L=2$ ). When using a MRC criterion based beamformer, installing more array elements assists us in obtaining a higher SNR gain, resulting in a better BER performance.

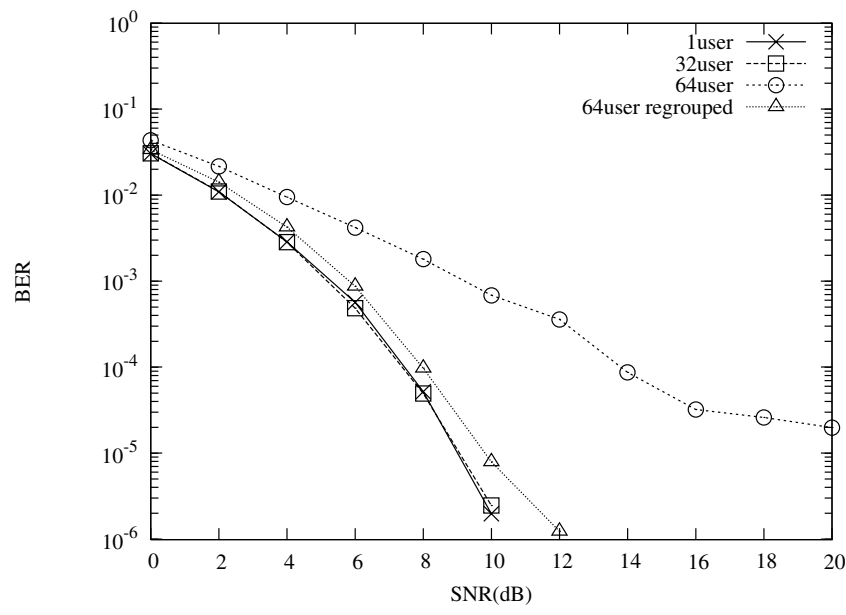

Fig. 4. BER versus SNR performance of the downlink of a generalized MC DS-CDMA wireless system using 32-chip Walsh-Hadamard (WH) codes as Tdomain spreading sequences and 4-chip WH codes as F-domain spreading sequences, using a $(2 \times 2)$-dimensional antenna array $(M=2, L=2)$. A SSTS scheme was used to pre-process the transmit signal. TF-domain spreading and user-grouping based on the interference coefficients were used to extend the capacity of the system, while suppressing the multiuser interference.

\section{CONCLusions}

In this contribution we have proposed a space-time transmitter processing scheme based on the principles of SSTS, in order to enhance the attainable performance of generalized MC DS-CDMA systems. Several different antenna array models have been investigated. Furthermore, generalized MC DS-CDMA using TF-domain spreading has been investigated and a user-grouping technique based on the interference coefficients was proposed for reducing the multiuser interference.

\section{REFERENCES}

[1] L. Hanzo, L. L. Yang, E. L. Kuan, and K. Yen, Single- and Multi-Carrier DS-CDMA. John Wiley \& Sons - IEEE Press, 2003, 1060 pages.

[2] L. Hanzo, M. Munster, B. J. Choi, and T. Keller, OFDM and MC-CDMA. John Wiley \& Sons - IEEE Press, 2003, 960 pages.

[3] L. Vandendorpe, "Multitone spread spectrum multiple access communications system in a multipath Rician fading channel," IEEE Transactions on Vehicular Technology, vol. 44, no. 2, pp. 327-337, 1995.

[4] E. A. Sourour and M. Nakagawa, "Performance of orthogonal multicarrier CDMA in a multipath fading channel," IEEE Transactions on Communications, vol. 44, pp. 356-367, March 1996.

[5] L.-L. Yang and L. Hanzo, "Performance of generalized multicarrier DSCDMA over Nakagami- $m$ fading channels," IEEE Transactions on Communications, vol. 50, pp. 956 - 966, June 2002.

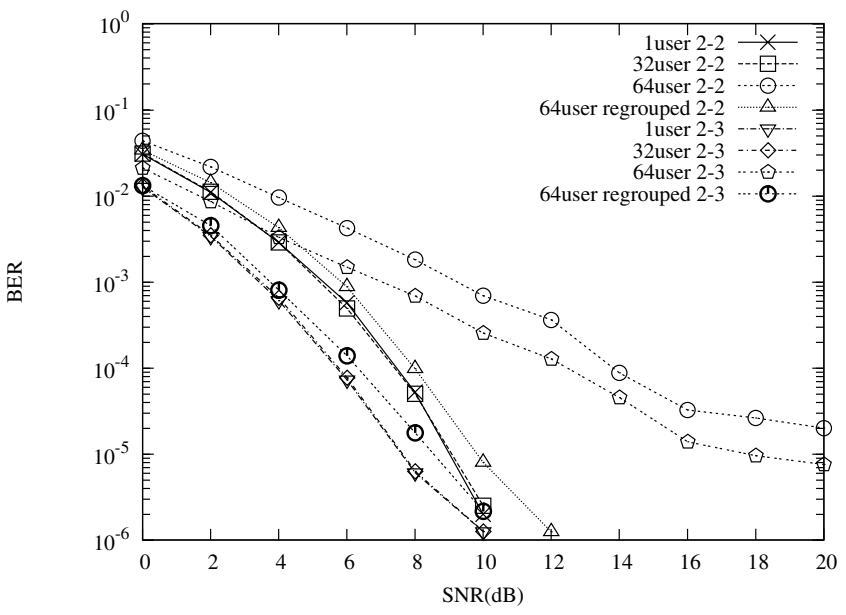

Fig. 5. BER versus SNR performance of the downlink of a generalized MC DS-CDMA wireless system using 32-chip WH codes as T-domain spreading sequences and 4-chip WH codes as F-domain spreading sequences. A SSTS scheme was used to pre-process the transmitted signal. TF-domain spreading was used to extend the capacity of the system, while an interference coefficient based user grouping technique was used to reduce the interference between the users sharing the same 32-chip WH code as T-domain spreading code. Two types of antenna arrays were employed, namely a $(2 \times 2)$-dimensional antenna array $(M=2, L=2)$, and a $(2 \times 3)$-dimensional antenna array $(M=2, L=$ $3)$.

[6] L.-L. Yang and L. Hanzo, "Performance of generalized multicarrier DSCDMA over using various chip waveforms," IEEE Transactions on Communications, vol. 51, pp. 748-752, May 2003.

[7] J. Blogh and L. Hanzo, Third-generation systems and intelligent wireless networking: smart antennas and adaptive modulation. John Wiley \& Sons - IEEE Press, 2002.

[8] J. H. Winters, "Optimum combining for indoor radio systems with multiple users," IEEE Transactions on Communications, vol. COM-35, pp. 1222-1230, Nov. 1987.

[9] D. Rajan and S. D. Gray, "Transmit diversity schemes for CDMA-2000," Proc. IEEE Wireless Communications and Networking Conf., pp. 669673, Sept. 1999.

[10] Proposed TDOC: $662 / 98$ to ETSI SMG2 UMTS Standards, Space-time block coded transmit antenna diversity for WCDMA, December 1998.

[11] Y. C. Liang and F. Chin, "Transmit antenna array techniques for celluar WCDMA systems," Proc. IEEE 9th PIMRC, pp. 1396-1400, Sept. 1998.

[12] Robert A. Soni and R. Michael Buehrer and Roger D. Benning, "Intelligent Antenna System for cdma2000," IEEE Signal Processing Magazine, pp. 54-67, July 2002.

[13] B. Hochwald and T. L. Marzetta and C. B. Papadias, "A Transmitter diversity scheme for wideband CDMA systems based on Space-time spreading," in IEEE Journal on Selected Areas in Communications, vol. 19, no. 1, pp. 48-60, Jan. 2001.

[14] L.-L. Yang and L. Hanzo, "Performance analysis of space-time spreading assisted wideband CDMA systems communicating over multipath Nakagami fading channels," in Submitted for Possible Publication (http://www-mobile.ecs.soton.ac.uk/lly), May, 2001.

[15] Ramjee Prasad and Shinsuke Hara, "Overview of Multicarrier CDMA," IEEE Communications Magazine, pp. 126-133, Dec. 1997.

[16] L.-L. Yang and L. Hanzo, "Space-Time Spreading Assisted Broadband MC DS-CDMA," in Proceedings of IEEE VTC'2002, Spring, (Birmingham, Alabama, USA), May 2002. 